\title{
PREPARACIÓN PROFESIONAL EN LA ATENCIÓN A LOS ESTUDIANTES CON DISCAPACIDAD DE LOS MAESTROS DEL SISTEMA GENERAL DE ENSEÑANZA ELEMENTAL EN PUERTO RICO
}

\author{
Professional preparation in the attention to the students with disabilities of the \\ teachers of the general system of elementary education in Puerto Rico
}

\section{Lilliam Vega}

Fecha de recepción: 12/09/2018

Fecha de aceptación: 10/12/2018

RESUMEN: La nueva era de los avances en la tecnología en programas asistivos mediante la computadora y la electrónica ha permitido que personas con discapacidades puedan ser independientes y trabajen en un gran número de áreas con los mismos derechos que todas y cada una de las personas en la sociedad. Los derechos adquiridos se pueden adjudicar a la Sección 1 del Artículo de la Constitución del Estado Libre Asociado de Puerto Rico que declara que la dignidad del ser humano es inviolable y establece que todos los seres humanos so $\mathrm{n}$ iguales ante la ley. Por consiguiente, el Gobierno de Puerto Rico tiene la responsabilidad indelegable de proteger, promover, defender, fomentar y crear las circunstancias particulares que propendan a la igualdad de vida para todos los miembros de nuestra sociedad. Desde la perspectiva legal, es importante que los administradores y los maestros consideren que estos hallazgos surgieren un posible incumplimiento con aquellas estipulaciones que establecen el derecho a una educación pública, gratuita y apropiada en el ambiente menos restrictivo, especialmente diseñada a las necesidades individuales de los estudiantes con discapacidad en la sala regular. Por tal razón, es necesario buscar nuevas alternativas que puedan ayudarnos a encontrar diversas maneras para trabajar con los servicios educativos y servicios relacionados en Asistencia Tecnológica (AT) en el currículo regular.

\section{PALABRAS CLAVES: Preparación, Conocimiento, Efectividad, Asistencia Tecnológica.}

ABSTRACT: The new era of advances in technology in computer and electronic assisted programs has allowed people with disabilities to be independent and work in a large number of areas with the same rights as each and every person in society. The acquired rights may be awarded to Section 1 of the Article of the Constitution of the Commonwealth of Puerto Rico stating that the dignity of the human being is inviolable and establishes that all human beings are equal before the law. Therefore, the Government of Puerto Rico has the nondelegable responsibility to protect, promote, defend, foster and create the circumstances that promote equality of life for all members of our society. From the legal perspective, it is important that administrators and teachers consider that these findings arise a possible noncompliance with those stipulations that establish the right to a public education, free and appropriate in the least restrictive environment, specially designed to the individual needs of the students. students with disabilities in the regular room. For this reason, it is necessary to look for new alternatives that can help us find different ways to work with educational services and related services in Assistive Technology (AT) in the regular curriculum.

KEY WORDS: Preparation, Knowledge, Effectiveness, Assistive Technology. 


\section{Introducción}

La nueva era de los avances en la tecnología en programas asistivos mediante la computadora y la electrónica ha permitido que personas con discapacidades puedan ser independientes y trabajar en un gran número de áreas con los mismos derechos que todos y cada una de las personas en la sociedad. Los derechos adquiridos se pueden adjudicar a la Sección 1 del Artículo 11 de la Constitución del Estado Libre Asociado de Puerto Rico que declara que la dignidad del ser humano es inviolable y establece que todos los seres humanos son iguales ante la ley. Por consiguiente, el Gobierno de Puerto Rico tiene la responsabilidad indelegable de proteger, promover, defender, fomentar y crear las circunstancias particulares que propendan a la igualdad de vida para todos los miembros de nuestra sociedad.

La Ley Pública 238 del 31 de agosto de 2004, conocida como la Carta de Derechos de las Personas con Discapacidades, resalta el reconocimiento de la condición de igualdad de todos los seres humanos en la Constitución, que impone al Gobierno del Estado Libre Asociado de Puerto Rico la responsabilidad indelegable de proteger, promover, defender, fomentar y crear las circunstancias particulares que propendan a la igual calidad de vida de todos los puertorriqueños y puertorriqueñas.

En Puerto Rico, existe una cantidad de 723,900 personas (Censo, 2010) que poseen uno o más discapacidades, esta cifra es equivalente a un 19.6 por ciento de 3,697,700 de la población de la isla. Esto implica que más de una cuarta parte de la población general necesita atención especial para alcanzar la plena calidad de vida y el desarrollo total de sus capacidades.

La Organización de las Naciones Unidas (ONU, 1999) reconoce que la educación es un derecho intrínseco y un medio indispensable para realizar otros derechos humanos. Esta debe orientarse al desarrollo de la personalidad humana, y a su vez capacitar a todas las personas para participar efectivamente en una sociedad libre. Debe favorecer la comprensión de los diferentes grupos en la sociedad. Por tal razón, la educación debe orientarse hacia el pleno desarrollo de la personalidad.

La Asistencia Tecnológica es todo equipo o servicio que pueda ser utilizado como herramienta por una persona con impedimentos, para aminorar el deterioro funcional, $\mathrm{o}$ aumentar y/o mantener capacidad funcional, o aumentar su nivel de capacidad para participar y recibir y una educación apropiada (Ley 51, 1996). Cuando hablamos de equipos de Asistencia Tecnológica nos referimos a que permiten que las personas con impedimentos se integren plenamente a la sociedad. Esto les faculta el mejorar su aprovechamiento académico o desempeño de funciones en el ambiente laboral. Dado el hecho que dichos equipos son por lo general costosos, debido a su carácter especializado, resulta necesario e indispensable, que exista un mecanismo que proteja la inversión de las personas con impedimentos que adquieren estos equipos (Ley 402, 2000).

En la actualidad se consideran las habilidades de las personas con discapacidades y se promueve la eliminación de las barreras arquitectónicas que le impiden alcanzar su máximo potencial y su inclusión en todos los contextos de la sociedad. Para llevar a cabo este proceso es indispensable poner a su disposición una gama de servicios educativos, tecnológicos y sociales que fomenten la inclusión de estos individuos en nuestra sociedad.

A su vez, el Departamento de Educación de Puerto Rico en cumplimiento con las disposiciones de la Ley Federal de Educación Elemental y Secundaria de 1965, según enmendada y reautorizada por la Ley No Child Left Behind (NCLB) del 2001, requiere que 
todos los maestros que enseñan una o más de las materias básicas en las escuelas públicas del país estén altamente cualificados. La definición aceptada por el Departamento de Educación de un maestro altamente cualificado es aquél que tiene, por lo menos, un bachillerato de una institución de educación superior acreditada, un certificado regular de maestro en la materia que enseña y ha demostrado competencia en cada una de las materias que enseña (Aragunde, 2005).

Por ende, los mandatos judiciales y las especulaciones prevalecientes en torno a la preparación de los maestros exigen que se investigue sistemáticamente la preparación profesional de los maestros del Sistema General de Enseñanza elemental sobre su percepción hacia el conocimiento y el uso efectivo de la Asistencia Tecnológica con los estudiantes con discapacidades y su aplicación en el currículo regular. Existe razón para creer que, particularmente la preparación de los maestros del Sistema General de Enseñanza y los maestros de Educación Especial hacia el uso efectivo de los equipos de Asistencia Tecnológica con los estudiantes con discapacidades, es extremadamente importante a la hora de aplicarlos en el currículo regular.

Con el transcurso de los años surgen nuevas leyes en defensa de los derechos de los niños y jóvenes con discapacidades, al recibir una educación libre, gratuita y apropiada, como lo establece la Ley Pública 94 -142 Education for all Handicapped Children Act de 1975. En la misma, el Congreso de los Estados Unidos, garantiza el derecho a una educación en el ambiente menos restrictivo para todos los niños de 3 a 21 años de edad.

En el 1986, el Congreso de los Estados Unidos aprobó la Ley Pública 99-447 conocida como Education for Handicapped Act Amendments, la cual le dio a Puerto Rico la oportunidad de establecer programas de intervención temprana para infantes y sus familias. En la Parte $\mathrm{H}$ de la ley se estipulan guías y requisitos para el desarrollo e implantación de servicios abarcadores, coordinados, multidisciplinarios e ínteragénciales de intervención temprana para infantes y bebés con discapacidades o rezago en el desarrollo y para sus familias. Esta ley enmienda la Ley Pública 94-142 Education for all Handicapped Children Act.

Es importante señalar los casos de corte que en sus comienzos fomentan la inclusión de los estudiantes con discapacidades en el currículo regular. En el 1954 el caso de corte Brown v. Board of Education of Topeka rompió la segregación racial en el sistema educativo de los Estados Unidos. Por primera vez se logró la integración de los estudiantes de raza negra con los estudiantes de este país. Con el transcurso de los años, en el 1986 Margaret Will's desarrolló el Modelo del REI (Regular Education Initiative) el cual se utiliza para adaptar la enseñanza -aprendizaje en el medio ambiente en todas las materias y permitir integrar a los estudiantes con discapacidades en el currículo regular.

La Ley IDEA de 1997, fue reautorizada el 4 de diciembre de 2004, por la Ley Pública 108- 446 Individuals with Disabilities Education Improvement Act . Esta ley establece que los Estándares Académicos deben estar alineados con lo expuesto en la Ley No Child Left Behind. Esto es, ofrecer a los estudiantes en el currículo regular el assessment; los maestros del Sistema General de Enseñanza deben estar altamente cualificados; el personal debe de: balancear disposiciones de disciplina, reducir el papeleo, mejorar las estrategias para intervención previa, reducir la sobre identificación e identificación errónea, entre otros.

El Congreso de los Estados Unidos realizó un análisis de los hallazgos de la Ley 10517 de 1997, Individuals with Disabilities Education Act ( IDEA) enmendada por la Ley 108446 del 4 de diciembre de 2004, Individuals with Disabilities Education Improvement Act ( 
IDEIA) sobre la educación especial presentado el 5 de enero de 2005, donde exponen que las discapacidades son una parte natural de la vida y no disminuyen el derecho del individuo a participar y contribuir en la sociedad. A su vez el mejoramiento de los resultados educativos para niños y jóvenes con discapacidades es un elemento esencial de la política nacional dirigida a asegurar igualdad de oportunidades, participación plena, vida independiente y autosuficiencia económica (Analysis of changes made by P.L. 108-446 Congressional research service, 2005).

Con esta investigación se pretende estudiar y analizar la preparación profesional de los maestros del Sistema General de Enseñanza elemental para explorar su preparación hacia el conocimiento y el uso efectivo de los equipos de Asistencia Tecnológica con los estudiantes con discapacidades y su aplicación en el currículo regular. De igual manera, la preparación es fundamental para tener maestros de excelencia con un conocimiento pleno en el área de especialidad, que fomenta la inclusión de todos los estudiantes con discapacidades utilizando la Asistencia Tecnológica en el currículo regular.

La experta realizó el estudio partiendo de las siguientes interrogantes:

1. ¿Qué conocimiento tiene el maestro del Sistema General de Enseñanza elemental sobre la Asistencia Tecnológica en el currículo regular?

2. ¿Qué preparación académica tiene el maestro del Sistema General de Enseñanza elemental hacia el uso efectivo de los equipos de Asistencia Tecnológica en el currículo regular?

3. ¿Cuál es el nivel de efectividad del uso de equipos y programas de Asistencia Tecnológica en el currículo regular?

\section{Enfoque del Marco conceptual}

El estudio de la preparación profesional de los maestros del Sistema General de Enseñanza elemental sobre su percepción hacia el conocimiento y el uso efectivo de los equipos de Asistencia Tecnológica con los estudiantes con discapacidades y su aplicación en el currículo regular, puede fundamentarse en varios marcos conceptuales.

Cook y Hussey (2002) determinaron que el diseñar diferentes sistemas de Asistencia Tecnológica es importante para entender los conceptos del desarrollo cognoscitivo y la relación que tienen en el uso de la tecnología para los estudiantes con discapacidades en el currículo regular. Cuando usamos el término aprendizaje nos referimos a los cambios que ocurren por el contacto con la influencia del medio ambiente. De igual manera, el término del desarrollo es una función en ambos como el crecimiento y el aprendizaje. Debemos de considerar que en el desarrollo del niño pueden ocurrir cambios significativos, lo cual es crucial para lograr que pueda utilizar la Asistencia Tecnológica, tanto en el hogar como en el currículo regular (Cook y Hussey, 2002).

Stainback y Stainback (1996) sustentaron que las escuelas están atemperadas en buscar las necesidades de todos los estudiantes para organizar una nueva estructura en las clases regulares que requerían unos cambios tales como el currículo, el personal de la escuela y los maestros. A su vez, estos cambios requerían una integración total para evitar la segregación de los estudiantes con discapacidades en el currículo regular. Cada una de las escuelas puede hacer uso del modelo de aprendizaje cooperativo como son las tutorías, la enseñanza de multiniveles y la integración curricular. 
Según estudios relevados por Puerto y otros (2000), los estudiantes que realizaron tareas ambientales en ambientes productivos experimentan una mayor motivación y responsabilidad. Es muy importante que los maestros provean estos ambientes y reciban a estos estudiantes en cursos ocupacionales porque producen un aumento de autonomía personal y social por la naturaleza de los mismos. Sin embargo, Howell (2001) indica que se espera que las escuelas y los maestros adopten estrategias apropiadas para acomodar a los estudiantes con discapacidades en el currículo regular.

De esta manera, Anderson y Hogan (2001) mediante una investigación de tipo exploratoria estudiaron los cambios en el conocimiento de los futuros maestros concerniente a la utilización de la tecnología en los estudiantes con discapacidades. Estos autores plantearon una serie de sugerencias para aumentar el conocimiento de los futuros maestros utilizando estrategias tales como, el exponer a estos futuros educadores a un adiestramiento combinado de clases demostrativas, discusiones grupales y actividades que fomentaron la investigación en los salones de clases, a la vez que promovieron más oportunidades en experiencias prácticas mediante la utilización de la Asistencia Tecnológica.

Goor (1995) coincide en afirmar que la administración de Educación Especial puede estar preparada para la iniciativa y manejo de AT porque beneficia a las personas con discapacidades, y es mandato de ley. La Nacional Council on Disabilities (1993) afirma que la AT es para personas con discapacidades para así obtener una educación apropiada en la escuela, reducir la dependencia con los miembros en la familia y maestros, economizar dinero, proveer empleos y mejorar la calidad de vida. Asimismo, la Ley 51 de 1996 define la Asistencia Tecnológica como todo aquel equipo indispensable que debe ser utilizado por personas con impedimentos con el propósito de aumentar, mantener, o mejorar sus capacidades funcionales. EL Office of Special Education Programs (OSEP) indica que en el distrito escolar es donde se determina las necesidades de AT para cada caso. Si en la reunión del Programa Educativo Individualizado (PEI) deciden que la AT es necesaria como parte de la educación del estudiante, el equipo y el servicio lo provee el distrito sin costo para los padres.

De igual manera, Mc Gray (2004) indicó que para implementar apropiadamente la Asistencia Tecnológica, los maestros deben poseer el conocimiento y las destrezas para tomar decisiones sobre la selección, implementación, y evaluación de la Asistencia Tecnológica utilizada (Lahm et. al., 2001). Por consiguiente, los miembros de la facultad deben explorar las formas de estructurar el currículo y las prácticas que mejor preparen al maestro para trabajar efectivamente con los estudiantes quienes utilizan equipos de AT (Byant, Erin, Lock, Allan y Resta, 1998). De esta manera, los autores recomiendan el uso de un plan de tecnología para facilitar la inclusión el cual es el primer paso para la preparación de los maestros y la evaluación de la tecnología en los programas de preparación del personal educativo. Los componentes de ese plan incluyen: una lista de los cursos que señalan la inclusión de la tecnología; los objetivos curriculares de tecnología; los procesos de evaluación; una lista de las herramientas tecnológicas necesarias en cada curso; y los servicios de apoyo necesarios, así como el personal para asistir a la facultad con los esfuerzos de inclusión de la tecnología.

Finalmente, la Asistencia Tecnológica continuará impactando y refinando la enseñanza emergente que involucra el uso de tecnología instruccional y asistiva para aumentar los resultados educativos relacionados a los estudiantes con discapacidades. 


\section{Población}

La población participante para el estudio formal fue proveniente de las diferentes escuelas del Distrito Escolar de Barranquitas y se seleccionó por disponibilidad de un subconjunto del universo donde representan esas características en la misma proporción. Fueron elegibles los maestros del Sistema General de Enseñanza elemental con experiencia en el magisterio de un año a treinta o más años.

La población de este estudio fue seleccionada por disponibilidad y que utilicen los equipos y materiales de Asistencia Tecnológica. Se seleccionó la Región Educativa de Caguas del Departamento de Educación de Puerto Rico que está compuesta por diez Centros de Desarrollo Profesional (Distritos Escolares) que son: Aguas Buenas, Aibonito, Barranquitas, Caguas I, Caguas II, Cayey, Cidra, Comerio, Guayama y Salinas. A su vez, se seleccionó el Centro de Desarrollo Profesional (Distrito Escolar) de Barranquitas para llevar a cabo el estudio debido a que es el que mayor cantidad de equipos y materiales de Asistencia Tecnológica solicitan en la Secretaría Asociada de Educación Especial del Departamento de Educación de Puerto Rico, para que los estudiantes con discapacidades utilicen estos en el currículo regular. Para seleccionar los participantes se considerará el criterio que los maestros del Sistema General de Enseñanza elemental estén ejerciendo funciones de maestros en el Sistema Educativo Público de Puerto Rico.

El Centro de Desarrollo Profesional (Distrito Escolar) que reúne todos los criterios de la muestra y desean participar de manera voluntaria es Barranquitas por la disponibilidad de los participantes, que ofrecen servicios de equipos y materiales de Asistencia Tecnológica. Este Centro se encuentra ubicado en la región centro de Puerto Rico. El mismo colinda por el área Norte con el pueblo de Orocovis, por el Sur con el pueblo de Coamo, por el Este colinda con el pueblo de Aibonito y en el área Oeste colinda con el pueblo de Jayuya.

\section{Muestra}

Los participantes considerados inicialmente para la muestra de este estudio se estimaron en 220 maestros del Sistema General de Enseñanza elemental, pertenecientes al Distrito Escolar de Barranquitas de la Región Educativa de Caguas. Finalmente, la muestra quedó configurada en un 55\% del total del Universo.

Los maestros tenían que contar con un año o más de experiencia como Maestros del Sistema General de Enseñanza elemental. Estos pertenecen a los grados desde kindergarten a sexto grado, que sirven a estudiantes con discapacidades integrados al Sistema General de Enseñanza elemental en las escuelas públicas del Sistema Educativo de Puerto Rico, específicamente del Centro de Desarrollo Profesional (Distrito Escolar) de Barranquitas, Puerto Rico.

Para seleccionar la muestra se utilizó un muestreo que consistió en sacar todos los casos $\mathrm{K}$ de una lista de la población (Ary, 1996). Este establece que cuando se conoce el número total de los miembros de la población $(\mathrm{N})$, se divide $\mathrm{N}$ entre $\mathrm{n}$ (muestra) para determinar el intervalo de $\mathrm{K}$ que se aplicará a la lista. El primer miembro se selecciona al azar entre los primeros miembros $\mathrm{K}$ de la lista y luego se escoge cada miembro $\mathrm{k}$ de la población para la muestra. Los casos K serán los maestros del Sistema General de Enseñanza pertenecientes al nivel elemental del Sistema General de Enseñanza del Distrito Escolar de Barranquitas de la Región Educativa de Caguas del Departamento de Educación de Puerto Rico. 


\section{Descripción del instrumento}

El instrumento que se utilizó en este estudio para recopilar los datos sobre Asistencia Tecnológica, efectividad, conocimiento, y preparación de los maestros del Sistema General de Enseñanza elemental fue desarrollado por la investigadora. Este cuestionario fue desarrollado basado en la revisión de la literatura relacionada con la preparación profesional de los maestros del Sistema General de Enseñanza elemental sobre su percepción hacia el conocimiento y el uso efectivo de los equipos de Asistencia Tecnológica con los estudiantes con discapacidades y su aplicación en el currículo regular. Los reactivos fueron construidos considerando las formas de estructurar el currículo y las prácticas que mejor prepara al maestro para trabajar efectivamente con los estudiantes quienes utilizan equipos de AT (Byant, Erin, Allan y Resta, 1998). Además, estos autores recomendaron los componentes de un plan de tecnología para facilitar la efectividad y evaluación de la tecnología en los programas de la preparación del personal educativo.

El instrumento que se utilizó para la recolección de datos de los maestros del Sistema General de Enseñanza elemental es: Preparación, Asistencia Tecnológica, Conocimiento y Efectividad (PACE). El instrumento se dividió en tres partes. La primera parte del instrumento está relacionada con aspectos demográficos de los participantes. La misma constó de nueve preguntas cerradas en las categorías distrito escolar, edad, género, último grado académico alcanzado, categoría del puesto que ocupa al presente, años de experiencia como maestros y tiempo que lleva en la escuela donde estuvo actualmente trabajando. Otro aspecto fue relacionado con los maestros que tenían estudiantes en la sala de clases utilizando programas y equipos de Asistencia Tecnológica. Por último, los maestros tenían que marcar con una (X) todas las categorías por discapacidades de estudiantes que impactan en el currículo regular. Es importante resaltar que apliquen por discapacidades basados en la Ley 51 y que la información esté incluida en el Programa Educativo Individualizado (PEI).

La segunda parte del instrumento consta de una escala Likert que se divide en tres secciones. La parte A midió el nivel de conocimiento en Asistencia Tecnológica que tiene el maestro del Sistema General de Enseñanza elemental hacia el uso efectivo de los equipos en el currículo regular. Esta parte consta de 8 reactivos. El participante seleccionó una de 5 respuestas en donde, el número 5 representa que posee mucho conocimiento y el número 1 representa ningún conocimiento.

La parte B del instrumento consta de 8 reactivos y midió el nivel de preparación de los maestros del Sistema General de Enseñanza elemental sobre la Asistencia Tecnológica que es utilizada en el currículo regular. El participante seleccionó una de 5 respuestas en donde, el número 5 representa totalmente de acuerdo y el número 1 representa totalmente en desacuerdo en tener la preparación en Asistencia Tecnológica.

La parte $\mathrm{C}$ del instrumento que midió el nivel de efectividad consta de 15 reactivos. El participante seleccionó una de 5 respuestas en la que el número 5 representa totalmente de acuerdo y el número 1 representa totalmente en desacuerdo, en torno a que existió un nivel de efectividad con respecto al uso de los equipos de Asistencia Tecnológica en el currículo regular.

La tercera parte del instrumento PACE, el grupo corresponde a la variable independiente Asistencia Tecnológica, el participante seleccionó con una (X) los equipos y programas de Asistencia Tecnológica que con mayor frecuencia utiliza cuando en su matrícula atienden estudiantes con diferentes discapacidades en la sala de clases regular. 
Baja Tecnología creadas por estudiantes universitarios en la formación de AT.

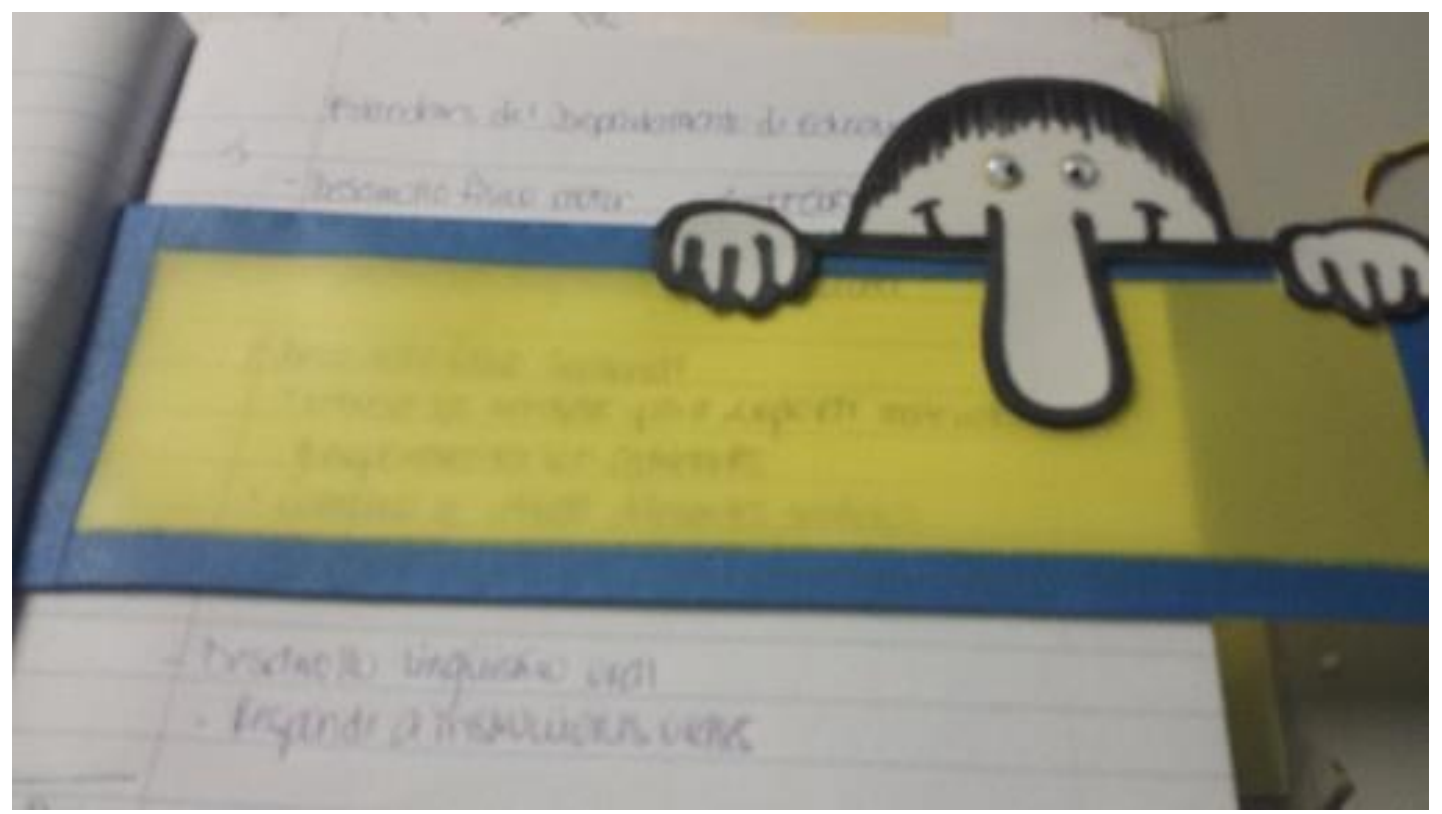

Enfoque Visual

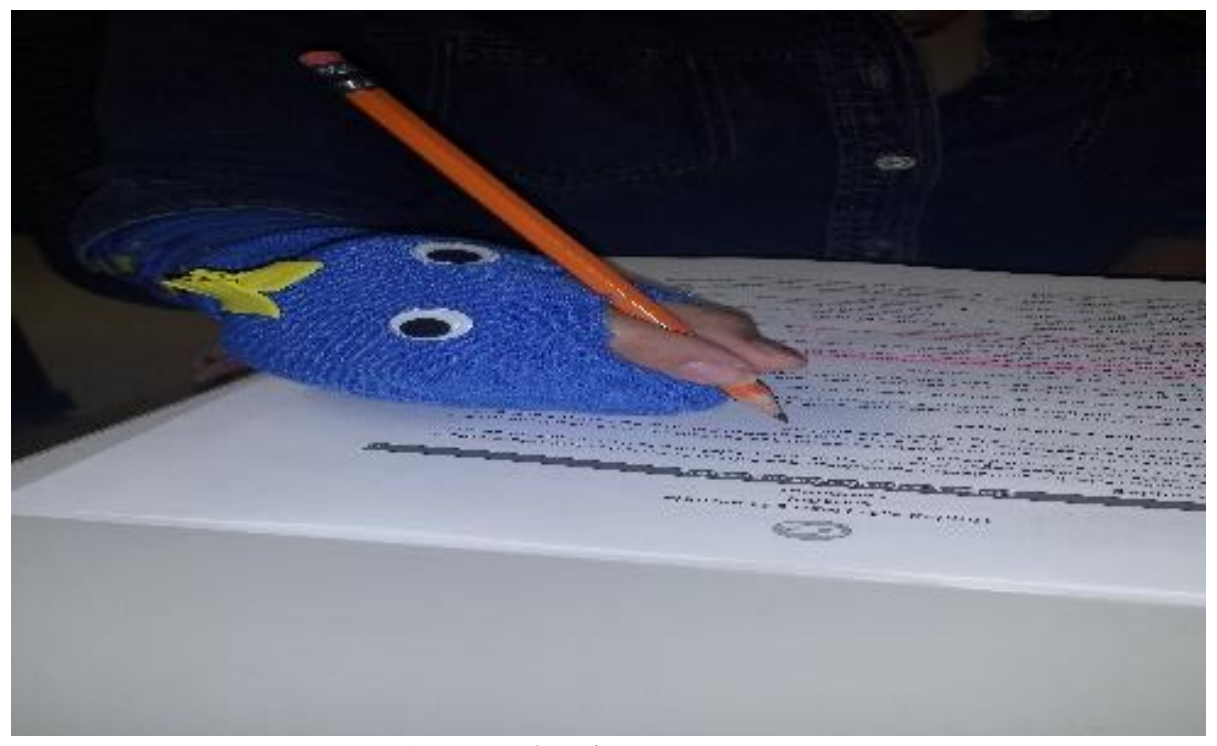

Agarre de pinzas

\section{Discusiones y conclusiones}

En Puerto Rico no se han realizado estudios dirigidos a examinar sobre la preparación de los maestros del Sistema General de Enseñanza elemental hacia el uso efectivo de los equipos de Asistencia Tecnológica en el currículo regular, que incluya de manera simultánea, un cuestionario que se desarrolla para medir las destrezas con el uso de equipos asistivos de los estudiantes con discapacidades sobre los temas que propone la investigación. Esta investigación tiene su importancia al amparo de las leyes de Asistencia Tecnológica, que establecen la necesidad de estudiar la preparación de los maestros del Sistema General de Enseñanza elemental hacia el uso efectivo de los equipos de Asistencia Tecnológica en el currículo regular. Los resultados de esta investigación pueden proporcionar a los 
administradores y educadores evidencia empírica sobre la preparación, el conocimiento, la Asistencia Tecnológica y la efectividad, el desarrollo profesional que poseen los maestros del Sistema General de Enseñanza elemental. Asimismo, pueden expandir el conocimiento de los maestros del Sistema General de Enseñanza elemental para aumentar las prácticas apropiadas de la implementación de la Asistencia Tecnológica en el proceso de enseñanza y aprendizaje.

El Departamento de Educación de Puerto Rico ha incurrido en gastos sumamente costosos en la compra de servicios de evaluaciones en Asistencia Tecnológica y equipo sofisticado para cubrir las necesidades de los estudiantes con discapacidades como lo estipulan las leyes vigentes. Esto constituye una prioridad dentro del sistema educativo, al identificar las alternativas apropiadas y eficientes para implantar un modelo de evaluación del progreso académico para los estudiantes con discapacidades que garantice el mejoramiento de la calidad de las prácticas educativas y el aumento en los logros de éstos.

A su vez es necesaria la Asistencia Tecnológica porque al surgir los cambios en la cultura social en nuestro medio ambiente promueve a que todos los estudiantes con discapacidades pueden estar integrados en el currículo regular. Esto toma un giro en las grandes ideas de los educadores, del cómo se agrupan a los estudiantes con discapacidades en el currículo regular, la toma de decisiones, las estrategias apropiadas, la percepción de los maestros hacia el uso efectivo de los equipos de Asistencia Tecnológica en la diversidad de estrategias de aprendizaje con variedad de destrezas en los diferentes niveles de ejecución. Por tal razón, los maestros deben buscar las alternativas de conocimiento para incluir los servicios suplementarios para cada uno de los estudiantes con discapacidades en el currículo regular. De igual manera, la efectividad y la Asistencia Tecnológica llega a ser un cambio de cultura social donde el enfoque es preparar a los maestros, facultad, administradores, padres, estudiantes, familiares, comunidad y apoyar a todos los estudiantes con discapacidades a tener mejor calidad de vida y actividad social a través de sus vidas.

Por ende, el fomentar el uso efectivo de los equipos de Asistencia Tecnológica es el trabajar en equipo colaborativo entre maestros, padres, estudiantes y administradores para proveerles a los estudiantes con discapacidades tener oportunidades de crecimiento en el medio ambiente con sus pares. En adición, los administradores tienen mayor influencia directa con los maestros hacia el uso efectivo de los equipos de Asistencia Tecnológica con los estudiantes con discapacidades en el currículo regular (Tripp y Rizzo, 2007). El énfasis hacia una educación de calidad para estudiantes con discapacidades requiere de altos estándares en términos de currículo, metas académicas y prácticas educativas que solo podrán ser alcanzados mediante una actitud positiva tanto dentro de la escuela como fuera de ella, creando un ambiente social integrado (Sage, 1995).

Los hallazgos de esta investigación proveen información a través de la percepción de los maestros del Sistema General de Enseñanza elemental sobre la preparación profesional, el conocimiento hacia el uso efectivo de los equipos de Asistencia tecnológica con los estudiantes con discapacidades y su aplicación al currículo regular.

Este estudio mostró que los maestros del Sistema General de Enseñanza elemental tienen bastante conocimiento para identificar una necesidad y referir a un estudiante con discapacidades para que este sea evaluado en Asistencia Tecnológica.

Los maestros del Sistema General de Enseñanza elemental tienen la necesidad de adquirir conocimiento y aplicar al currículo regular para los estudiantes con discapacidades. 
Por consiguiente, estarán dispuestos a conocer y a utilizar los equipos y programas de Asistencia Tecnológica en la sala de clases regular.

Se pueden establecer estrategias para desarrollar bancos de recursos que puedan colaborar en talleres de capacitación en preparación de equipos de baja tecnología en todas las materias académicas.

Por tal razón, los maestros del Sistema General de Enseñanza elemental deben capacitarse en dominar los equipos de alta tecnología para cuando tengan que trabajar con un estudiante con discapacidades en la sala de clases regular.

Es preocupante estos resultados porque afirma que los maestros del Sistema General de Enseñanza elemental deben tener la preparación y el conocimiento para ofrecer a los estudiantes con discapacidades las herramientas necesarias para lograr el éxito en el ámbito educativo dentro del Departamento de Educación de Puerto Rico.

Los maestros del Sistema General de Enseñanza elemental deben estar asertivos a los cambios en la evolución de los adelantos tecnológicos ya que estos permiten un aprendizaje más efectivo en igualdad de condiciones para los estudiantes con discapacidades visuales en el currículo regular.

Es necesario que los maestros del Sistema General de Enseñanza elemental se capaciten para poder asignar tareas a los estudiantes con discapacidades.

Cuando se ofrecen adiestramientos al personal no es suficiente porque hay que evaluar la aplicación del conocimiento y observar los resultados obtenidos en un tiempo determinado. Si resultan cambios significativos hay que retomar la información y realizar los arreglos necesarios para mejorar la efectividad de lo aprendido.

La presente investigación pretende aportar y concienciar la preparación profesional de los maestros del Sistema General de Enseñanza elemental sobre su percepción hacia el conocimiento y el uso de los equipos de Asistencia Tecnológica con los estudiantes con discapacidades y su aplicación en el currículo regular. Esta intenta ser una propuesta de reflexión y acción para los administradores de la Secretaría Asociada de Educación Especial del Departamento de Educación de Puerto Rico, y sobre todo, para los supervisores, directores y maestros implicados en atender las necesidades de los estudiantes con discapacidades en la sala de clase regular.

Johnston y otros (2007) determinaron que cuando los niños con discapacidades han tenido dificultades pueden adquirir diferentes apoyos que pudieran ser implementados para su beneficio personal. De igual manera, la Asistencia Tecnológica llega a tener un significado contundente para ayudar a los niños a alcanzar la independencia. Asimismo, la efectividad de los equipos o servicios de AT pueden ser una expectativa favorable en cuanto al crecimiento de los niños, su desarrollo, su maduración y que sus necesidades pueden cambiar con el tiempo.

A continuación, se exponen algunas implicaciones relacionadas con este estudio, para la Secretaría Asociada de Educación Especial, para el distrito escolar, las escuelas y las universidades. 


\section{Secretaría Asociada de Educación Especial}

En la Secretaría Asociada de Educación Especial se debe concienciar a los administradores, a los supervisores y a otro personal que esté adscrito a ofrecer servicios relacionados con la Asistencia Tecnológica para los estudiantes con discapacidades. Por consiguiente, se debe crear una Unidad de Asistencia Tecnológica dirigida por un director (funcionario) que tenga el peritaje en esta área y trabajar con un equipo de personas adiestradas o certificadas en Asistencia Tecnológica. Esto se refiere específicamente, al ofrecimiento en el sistema educativo, reciban los servicios de otros especialistas del Programa de Asistencia Tecnológica de Puerto Rico (PRATP).

La solución para esta situación es tan alarmante en la actualidad de nuestro sistema educativo es certificar al personal necesario para que puedan evaluar a los estudiantes con discapacidades y evitar las querellas que diariamente ocasionan penalidades debido a las demandas de clase en la corte. Es necesario además, que la compra de los programados y equipos de Asistencia Tecnológica con los respectivos adiestramientos y las garantías requeridas, conlleva un servicio aún cuando caduquen, para que los estudiantes con discapacidades, los maestros y los padres puedan utilizarlos con efectividad en la sala de clases y el hogar.

\section{Distrito escolar}

En el distrito escolar, el Superintendente de escuelas, Facilitador de Educación Especial con el apoyo del personal de la Unidad de Asistencia Tecnológica de la Secretaría Asociada de Educación Espacial pueden coordinar las certificaciones en el uso de los programados de Asistencia Tecnológica para los maestros de Tecnológica Educativa y los maestros de Educación Especial. De esta manera los maestros del Sistema General de Enseñanza pueden atender las necesidades y mejorar el aprovechamiento académico de los estudiantes con discapacidades en el currículo regular.

\section{Escuelas}

En las escuelas del sistema público de Puerto Rico se puede implantar como proyecto piloto en el nivel elemental el que los maestros bibliotecarios en la biblioteca puedan tener accesibles computadoras con los programados de Asistencia Tecnológicas necesarios para atemperar las necesidades de los estudiantes con discapacidades en el currículo regular. A su vez, puedan ser utilizados por la comunidad en el horario extendido de la escuela.

\section{Universidades}

Las universidades se dirijan a preparar maestros, especialistas o personal interesado en las certificaciones de Asistencia Tecnológica y Maestrías o Doctorados en Tecnología e Innovación Educativa, Inteligencia Artificial con especialidad en Asistencia Tecnológica.

\section{Limitaciones}

Esta investigación es la primera que se realiza en Puerto Rico sobre la preparación profesional de los maestros del Sistema General de Enseñanza elemental sobre su percepción hacia el conocimiento y el uso efectivo de los equipos de Asistencia Tecnológica con los estudiantes con discapacidades y su aplicación en el currículo regular, en el Departamento de Educación. 
1. La muestra del estudio se circunscribió a las escuelas elementales del pueblo de Barranquitas. Esto generó actitudes poco usuales en la prestación del interés por parte de los maestros del Sistema General de Enseñanza elemental de dicho pueblo al recopilar la muestra.

2. Los resultados en este estudio describen la preparación profesional de los maestros del Sistema General de Enseñanza elemental y no necesariamente reflejan la realidad del proceso en todas las escuelas de Puerto Rico.

3. Es necesario realizar más investigaciones sobre los hallazgos para visualizar la extensión en que estos convergen en otros distritos escolares.

\section{Recomendaciones para futuras investigaciones}

Este estudio provee una descripción del nivel de preparación profesional, del conocimiento, de la efectividad y del uso de la Asistencia Tecnológica por parte del maestro en el nivel elemental del currículo regular. La reautorización de la Ley IDEA y de No Child Left Behind garantiza que estos estudiantes deben ser considerados en todas las actividades desarrolladas para mejorar los resultados educativos.

La investigadora hace las siguientes recomendaciones para futuras investigaciones:

1. Realicen estudios similares en otros pueblos y regiones para auscultar si existen diferencias significativas entre su percepción hacia el conocimiento y el uso efectivo de los equipos de Asistencia Tecnológica con los estudiantes con discapacidades y su aplicación al currículo regular.

2. Se lleven a cabo investigaciones posteriores en los niveles intermedios y superiores para establecer si existen diferencias significativas entre las variables utilizadas en esta investigación.

3. Promueva un estudio focal para identificar estrategias y actividades que propicien mejorar la participación de los maestros del Sistema General de Enseñanza elemental en el uso efectivo de los equipos de Asistencia Tecnológica y su aplicación al currículo regular.

4. Ejecuten nuevos estudios donde se utilicen investigaciones recientes y nuevos marcos teóricos, sobre la preparación profesional de los maestros del Sistema General de Enseñanza elemental sobre su percepción hacia el conocimiento y el uso efectivo de los equipos de Asistencia Tecnológica y su aplicación a currículo regular.

5. Investiguen estudios cuasi experimentales con pre-prueba y post- prueba con el propósito de conocer la preparación profesional de los maestros del Sistema General de Enseñanza elemental antes y después de implementar adiestramientos sobre ventajas y desventajas del uso de efectivo de los equipos de Asistencia Tecnológica y su aplicación al currículo regular. 


\section{Referencias bibliográficas}

Acta de Asistencia Tecnológica. (1998). Ley Núm. 108-364 enmendada en el 2004, San Juan, Puerto Rico.

Agencia EFE. (17 de abril de 2017). 286,000 personas en la isla tienen algún impedimento. El Nuevo Día. Recuperado de:

https://www.elnuevodia.com/noticias/locales/nota/286000personasenlaislatienenalguni mpedimento-2311983/.

Analysis of changes made by P.L. 108-446 Congressional research service. (2005). Individuals with disabilities education act (IDEIA) The library of congress. Department of Education of United State.

Americans with Disabilities Act. Ley Núm. 101-336 de 1990, Unite State.

Anderson, C. L. y Borthwick, A. (2002). Results of separate and integrated technology instruction in pre-service training. National Educational Computing Conference Proceedings, 06, 1-54. Recuperado de:

http://www.eric.ed.gov/ERICWebPortal/Home.portal?_nfpb=true\&_page Label=ERICSearchResult.

Anderson, C. L. y Hogan, B. P. (2001). The impact of technology use in special education field experience on pre-service teachers' perceived technology expertise. Journal of Special Education Technology, 16, 1 - 43. Recuperado de: http://jset.unlv.edu./16.3/issuemenu.html.

Andrews, L. (2002). Preparing general education pre-service teachers for inclusion: web-enhanced case-based instruction. Journal of Special Education Technology, 17, 1 -30. Recuperado de: http://jset.unlv.edu./17.3/issuemenu.html.

Aragunde, R. (2005). Notificación de la disponibilidad de instrumento evaluación estándar nacional altamente objetiva y uniforme para evidenciar competencia profesional de maestros que no cumplen con el requisito de altamente cualificados de la ley pública 107-110, sección 1111 (h) (a) y (b).

Departamento de Educación de Puerto Rico.

Ary, D., Jacobs, L. Ch. y Razavieh, A. (1996). Introducción a la investigación pedagógica. México: MacGraw-Hill.

Ayvazoglu, N. R., Oh, H., y Kozub, F. M. (2006). Explaining activity in children with visual impairment: a family systems approach. Journal of Exceptional Children, 72, 235-246.

Bain, B. y Leger, D. (1997). Assistive technology: an interdisciplinary approach. New York: Churchill Livingstone, Inc.

Barnes, S. y Whinnery, K. W. (2003). Effects of functional mobility skills training for young students with physical disabilities. Journal of Exceptional Children, 68, 313-323. Recuperado de: 
http://www.questia.com/PM.qst?action=print\&docld=5001705006.

Blankership, T., Ayres, K. y Langone, J. (2005). Effects of computer-

based cognitive mapping on reading comprehension for students with emotional behavior disorders. Journal of Special Education Technology, 20, 15-23.

Bermann, M.M. (1995). Assistive technology for students with mild disabilities. Council for Exceptional Children, 30, 70-83.

Recuperado de:

http://www.eric.ed.gov/ERICDocs/data/ericdocs2/content.storage_01/000.

Best, J. W. (1982). Cómo investigar en educación. (Novena edición).

Madrid: Ediciones Morata, SA.

Bolck, M. E. (1994). A teachers guide to incluiding students with disabilities in regular physical education. Baltimore, MD.: Paul H. Brookes.

Cain, H. y Zandria, M. (2001). Distance education for master's students with visual impairments: Technology and support. Journal of Visual Impairment \& Blindness, 95, 572 - 579. Recuperado de: http://www.eric.ed.gov/ERICWebPortal/Home.portal?_nfpb=true\&_pageLabel=ERIC SearchResult\&_urlType=action.

Carl D. Perkins. Ley Núm. 105-332 enmendada el 31 de octubre de 1998, San Juan, Puerto Rico.

Carta de Derechos de las Personas con Discapacidades. Ley Núm. 238 del 2004. San Juan, Puerto Rico.

Constitución del Estado Libre Asociado de Puerto Rico. Ley Núm. 201, San Juan, Puerto Rico.

Castle, E.D. (1967). Ancient education and today. Baltimore, Md. Penguin Books.

Cook, A. M. y Hussey, S.M. (2002). Assistive technologies principles and practice. St. Louis, Missouri, USA: Mosby, Inc.

Copley, J. y Ziviani, J. (2004). Barriers to the use of assistive technology for children with mental retardation. Division of Occupational Therapy University of Queensland, St. Lucia, Australia.

Cowan, M. y Khan, Y. (2005). Assistive technology for children with moderate mental disabilities. Journal of Development and Physical Disabilities, 15, 207-212.

Davis, M., Robins, B., Dautenhahn, K., Nehsniv, C. y Powell, S. A. (2005). A comparison of interactive and robotic systems in therapy and education for children with autism. Adaptive Systems Research Group, University of Hertfordshire, UK, Department of Education, University of Hertfordshire, 
Recuperado de: http://www.aurora-project.com.

De Landsheere, G. (1985). Diccionario de la evaluación y de la investigación educativas. Barcelona, España: Oikos-tau, SA.

Departamento de Educación y Administración de Veteranos. (1972). Nacional Institute on Handicapped Research. USA.

Departamento de Educación de Puerto Rico: Carta Circular Núm. 20-2003-2004 del 30 de junio de 2004. Directrices para la implantación de la autonomía fiscal, administrativa y docente de la Secretaría Auxiliar de Servicios Educativos Integrales para Personas con Discapacidades, San Juan, Puerto Rico: autor.

Departamento de Educación de Puerto Rico: Carta Circular Núm. 5-2004-2005 del 3 de septiembre de 2004. Manual de Procedimientos de Educación Especial, San Juan, Puerto Rico: autor.

Departamento de Educación de Puerto Rico: Carta Circular Núm. 4-87-88 de enero de 1988, Normas y procedimientos para el ofrecimiento de Servicios de Educación Especial a los niños y jóvenes con discapacidades, San Juan, Puerto Rico: autor.

Departamento de Educación de Puerto Rico. (2003). Proyecto de renovación curricular: Fundamentos teóricos y metodológicos. San Juan, Puerto Rico: Publicaciones Puertorriqueñas.

Diccionario práctico del estudiante. (2007). Real Academia Española, Asociación de Academias de la Lengua Española. Santillana, Madrid: España. Recuperado de: http://www.rae.es/.

Distrito Escolar de Barranquitas (2007). Región Educativa de Caguas. Departamento de Educación de Puerto Rico.

Education of the Handicapped Act. Ley Núm. 99-457 de 1986, USA.

Employment Opportunity for Disabled Americans. Ley Núm. 99-463 de 1986, USA.

Enciclopedia de investigación educativa. (1992). (6ta. ed.) I-II. Editor Marvin C. Alkin. New York: Mc Millan.

Erickson, W., Lee, C. y Von Schrader, S. (2012). 2010 Reporte del estado de la discapacidad: Puerto Rico, Estados Unidos. New York: Cornell University: Instituto de Empleo y Discapacidad (EDI).

Erlanger, H. y Roth, W. (1985). Disability policy: the parts and the whole. American Behavioral Scientist, 28, 319-346.

Falcón, L.E. (2001). El nivel de efectividad y satisfacción de consumidores y consejeros sobre los servicios y el uso de la asistencia tecnológica en la administración de Rehabilitación Vocacional. Disertación doctoral no publicada, Interamericana, San Juan, Puerto Rico.

Galvin, J. y Scherer, M. (1996). Evaluating, selecting and using appropriate 
assistive technology. Maryland: Aspen Publishers.

Garantías sobre equipos de Asistencia Tecnológica de Puerto Rico. Ley Núm. 402 de 2000, San Juan, Puerto Rico.

González, J.A. (1999). Conocimiento, opinión y actitud de los maestros de la sala Regular y de educación especial hacia la asistencia tecnológica como parte del proceso de enseñanza-aprendizaje de los estudiantes con discapacidades. Disertación doctoral no publicada, Interamericana, San Juan, Puerto Rico.

Guajardo, E. (1999). La inclusión e integración educativas en el mundo.

Implicaciones teóricas metodológicas y sociales. Revista de Educación nueva época 11, 1- 10. Recuperado de:

http://educacion.jalisco.gob.mx/consulta/educar/11/11indice.html.

Hamm, E. M., Mistrett, S. G. y Goetz Ruffino, A. (2006). Play outcomes and Satisfaction with toys and technology of young children with special needs. Journal of Special Education Technology, 21, 29-35.

Hernández Santos, E. (1994). Diccionario fundamental de la educación.

San Juan, Puerto Rico: Publicaciones Puertorriqueñas

Hernández Sampieri, R., Fernández Collado, C., y Baptista Lucio, P. (2010). Metodología de la investigación. (5ta. ed.) México: Mc Graw-Hill.

Hider, E.D. (2000). A qualitative study of the child, family and professional factors that influence the use of assistive technology in early intervention. Capitalizing on Leadership in Rural Special Education: Making a Difference for Children and Families, 03, 79-85. Recuperado de: http://www.eric.ed.gov/ERICWebPortal/Home.portal?_nfpb=true\&_pageLabel=ERIC SearchResult\&newSearch=true.

Hilt, A. y Rosethal, D. (2004). A method for conditioning reinforces preferences in students with moderate mental retardation. Journal of Developmental and Physical Disabilities, 16, 32-50.

Howell, R. T. (2001). Industrial technology education teacher's knowledge, experience and feelings related to working with special population students in the Lincoln, Nebraska Public School. Journal of Industrial Teacher Education, 38, 2-11.

Individuals with Disabilities Education Act, IDEA. (1990). Ley Núm. 105-17 de 1997, USA.

Individuals with Disabilities Improvement Act (IDEIA).

Ley Núm. 108-446, 4 de diciembre de 2004, USA.

Isaac, S. y Michael, W. B. (1993). Handbook in research and evaluation. California: Edits Publishers.

Khun, T. (1962). The structure of scientific revolutions. Madrid, España. 
Lizama, M. A. y Méndez, H. L. (1995). Assistive technology development in Puerto Rico. Annual Florida Assistive Technology Impact Conference \& Conference Of the TAM Division of the Council for Exceptional Children, 141, 1-41. Recuperado de: http://www.eric.ed.gov/ERICWebPortal/Home.portal? $\_n f p b=$ true\&_pageLabel=ERICSearchResult\&newSearch=true.

Llorens, S. (1992). Significado y actitudes de la formación continua: Un estudio cualitativo con el sector metal-mecánica. Universitat Jaume I de Castellón, Valencia: España. Recuperado de: http://www.uji.es/bin/publ/edicions/jfi3/signif.pdf\#search=\%22actitudes $\% 20 \% 2 \mathrm{~B} \% 20$ significado $\% 22$.

Manual de Procedimientos de Educación Especial. (2004). Departamento de Educación de Puerto Rico, San Juan, Puerto Rico: autor.

Manual de procedimientos de servicios asistencia tecnológica. (2001).

Departamento de Educación de Puerto Rico, San Juan Puerto Rico: autor.

Massone, M. I., Buscaglia, V. L., y Bogado, A. (2005). Los sordos aprenden a escribir sobre la marcha. Lectura y Vida, Año XXVI, 4, 6-17.

Maushak, N. J., Kelley, P. y Blodgett, T. (2001). Preparing teachers for the inclusive classroom: A preliminary study attitudes and knowledge of Assistive Technology. Journal of Technology and Teachers Education, 9, 419 - 431. Recuperado de: http://www.eric.ed.gov/ERICWebPortal/Home.portal?_nfpb=true\&_pageLabel=ERIC SearchResult\&newSearch=true.

Medicaid Amendment for Special Education Related Services. Ley Núm. 100-360 de 1988, USA.

Medical Early and Periodic Screening, Diagnosis and Treatment Amendments. Ley Núm. 101-238 de 1989, USA.

Michaels, C.A. y McDermott, J. (2003). Assistive technology integration in special education teacher preparation: Program coordinators' perceptions of current attainment and importance. Journal of Special Education Technology, 18, 1 - 20. Recuperado de: http://jset.unlv.edu./18.3/issuemenu.html.

Ochoa, T. A., Kelly, M. L., Stuart, S. y Rogers Adkinson, D. (2004). The impact of PBL technology on the preparation of the teachers of english language learners. Journal of Special Education Technology, 19, 1-16. Recuperado de: http://jset.unlv.edu./19.3/issuemenu.html.

Moreno, J. (1978). Historia de la educación. Madrid. Paraninfo.

No Child Left Behind. Ley Núm. 107-110 del 2001, USA.

Orgánica para el Departamento de Educación Pública de Puerto Rico. 
Ley Núm. 149 del 15 de julio de 1999, San Juan, Puerto Rico.

Ortiz García, A. L. (2003). Diseño y evaluación curricular. Río Piedras, Puerto Rico. Editorial Edil, Inc.

Ozmon Howard, C. (1981). Philosophical foundations of education.

Columbus, Ohio, Charles Merrill.

Piaget, J. (1972). Foco de estudio y procesos de aprendizaje de Piaget.

Recuperado de:

http://campus.uab.es/ 2133542/teoriap.html.

Pounds, R. (1968). The development of education in western culture. N. Y. Appleton- Century-Crofts.

Programa de Asistencia Tecnológica de Puerto Rico. Ley Núm. 264 de 2000, San Juan, Puerto Rico.

Programa de Asistencia Tecnológica de Puerto Rico, PRATP (2005).

Recuperado de: http://pratp.upr.edu/defiat.htm.

Puerto, M. y Valladolid, E., Laserna, J.L., y Gracia, A. (2000). Aula puente: Transición de las personas con necesidades educativas especiales de la escuela al trabajo. Recuperado de: http://centros6.pntic.mec.es/ primitiv/aulapuente.htm.

Quinn, M. M., Rutherford, R. B., y Osher, D. M. (1999). Special education in alternative Education programs. Recuperado de: http://www.ericdigests.org/2003/special.htm.

Rehabilitation Act. Ley Núm. 95-602 de 1978, USA.

Rehabilitation Act. Ley Núm. 99-506 de 1996, USA.

Reglamento de Certificación del Personal Docente de Puerto Rico. (2003). Departamento de Educación de Puerto Rico.

Roessler, R. y Bolton, B. (1978). Phychological adjusment to disability. Baltimore, MD : University Park Press.

Roulstone, A. (1998). Enabling technology: disabled people, work and new technology. Open University Press, Philadelphia, PA.

Sage, D. (1995). Inclusion times. The National Profesional Resources, Inc., 25 South Regent St., Port Chester, NY 10573.

Sage, D. D. y Burrello, L.C. (1994). Leadership in educational reform.

Maryland: Paul H. Brookes Publishing Co., Inc., British Library.

Santos y Vargas. L. (1992). Crítica filosófica de la educación: escenario 
puertorriqueño. San Juan, Puerto Rico: Publicaciones Interdisciplinarias, Universidad de Puerto Rico.

Scherer, M., y Lane, J. (1997). Assesing consumer profiles of ideal assistive Technologies in ten categories: an integration of quantitative methods. Disability and Rehabilitation, 19, 528-535.

Servicios Educativos Integrales para Personas con Discapacidades.

Ley Núm. 51 del 7 de junio de 1996, San Juan, Puerto Rico.

Sherrill, C. (1998). Adapted physical activity, recreation and sport: Cross disciplinary and life spam. Madison, WI : WCB. MacGraw-Hill.

Smith-Fess Act. Ley Núm. 67-236 de 1920, USA.

Social Security Act, Ley Núm. 74-271 de 1935, USA.

Social Security Act, Ley Núm. 84-880 de 1956, USA.

Social Security Act, Ley Núm. 89-97 de 1965, USA.

Special Education Inclusion. Recuperado de: http://www.weac.org/resource/june96/speced.htm.

Stainback, W. y Stainbck, S. (1996). Controversial issues confronting special education divergent perspectives. New Jersey, USA: Allyn \& Bacon.

Technology-Related Assistance for Individuals with Disabilities (Tech Act). Ley Núm. 103-218 de 1994, USA.

Teoría del conocimiento es una doctrina filosófica. (1997).

Recuperado de:

http://www.monografias.com/trabajos/epistemologia2/epistemologia2.shtm.

Torres Serrano, L. M. (1999). Asistencia tecnológica: una posibilidad real.

San Juan, Puerto Rico: Editorial Isla Negra, Búho.

Torres Serrano, L. M. (2000). Estrategias de intervención para la inclusión.

San Juan, Puerto Rico: Editorial Isla Negra, Búho.

UNESCO (1945). Recuperado de: http://portal.uneco.org/es/ev.phpURL_ID+328\&URL_DO+DO_TOPIC\&URL_ SECTI.

Vocational Rehabilitation Amendments Act. Ley Núm. 113 de 1943, USA.

Vocational Rehabilitation Act. Ley Núm. 82-565 de 1954, USA.

Vocational Rehabilitation Act. Ley Núm. 89-333 de 1965, USA. 
Wallace, J., Flippo, K., Barcus, J. y Berhmann, M. (1995). Legislative foundation of assistive technology policy in United States. In K. Inge, \& M. Barcus (Eds.), Assistive technology: a resource for school, work and community. Maryland: Paul H. Brookes.

Wang, L. y Ertmer, P. A. (2003). Impact of vicarious learning experiences and goal setting on pre-service teachers' self-efficacy for technology integration: A pilot study. Paper presented at the Annual Meeting of the American Educational Research Association, 00, 1-15. Recuperado de:

http://www.eric.ed.gov/ERICWebPortal/Home.portal?_nfpb=true\&_page Label=ERICSearchResult.

Warger, C. (1998). Integrating assistive technology into the standard curriculum. ERIC/OSEP Digest, 08, 1-4. Recuperado de:

http://www.eric.ed.gov/ERICWebPortal/Home.portal?_nfpb=true\&_pageLabel=ERIC SearchResult\&newSearch=true.

Will, M.C. (1996). Educating childrens with learning problems: A shared responsibility. Washington, DC, USA. Department of Education. Office of Special Education and Rehabilitation Services.

Wyatt, C. (2003). Assistive technology aids teenager with mental retardation to Gain mealtime independence. Mealtime Partners, Inc. Parkway Azle, TX. 